\title{
Mandibular Midline Distraction Osteogenesis with a Bone-borne, Tooth-borne or Hybrid Distraction Appliance: a Systematic Review
}

\author{
Thomas Starch-Jensen ${ }^{1}$, Annette Dalgaard Kjellerup ${ }^{1}$, Tue Lindberg Blæhr ${ }^{1}$ \\ ${ }^{1}$ Department of Oral and Maxillofacial Surgery, Aalborg University Hospital, Aalborg, Denmark.
}

\author{
Corresponding Author: \\ Thomas Starch-Jensen \\ Department of Oral and Maxillofacial Surgery \\ Aalborg University Hospital \\ 18-22 Hobrovej, DK-9000 Aalborg \\ Denmark \\ Phone: +4597662798 \\ Fax: +4597662825 \\ E-mail: thomas.jensen@rn.dk
}

\begin{abstract}
Objectives: The objective of the present systematic review was to assess the transverse skeletal and dental arch expansion and relapse after mandibular midline distraction osteogenesis with a bone-borne, tooth-borne or hybrid distraction appliance. Material and Methods: A MEDLINE (PubMed), Embase and Cochrane library search in combination with a hand-search of relevant journals was conducted. Human studies published in English until the $3^{\text {rd }}$ of July, 2018 were included.

Results: Two comparative and seven non-comparative studies characterized by high risk of bias fulfilled the inclusion criteria. Transverse mandibular widening was achieved with the different types of distraction appliance displaying a horizontal V-shaped opening with larger anterior transverse expansion declining progressively towards the posterior part of the mandible. Boneborne and hybrid appliance facilitate more skeletal expansion compared with tooth-borne appliance, whereas comparable dental arch expansion was achieved with the different types of distraction appliance. Skeletal and dental arch relapse with the different type of appliance was limited and comparable. However, frequency of complications was higher with bone-borne appliance compared with tooth-borne or hybrid appliance.

Conclusions: Mandibular midline distraction osteogenesis with bone-borne, tooth-borne or hybrid distraction appliance is an effective treatment modality to correct severe transverse mandibular discrepancies, although the skeletal and dental arch expansion pattern was dissimilar with the different types of appliance. However, dissimilar evaluation methods, different outcome measures, various methodological confounding factors posed serious restrictions reviewing the literature in a quantitative systematic manner. Hence, well-designed long-term randomized controlled trials applying three-dimensional technology, patient-related outcome measures and an economic perspective are needed before definite conclusions can be provided.
\end{abstract}

Keywords: bone lengthening; mandible; orthodontics; orthognathic surgery; review.

Accepted for publication: 28 September 2018

To cite this article:

Starch-Jensen T, Kjellerup AD, Blæhr TL.

Mandibular Midline Distraction Osteogenesis with a Bone-borne, Tooth-borne or Hybrid Distraction Appliance: a Systematic Review

J Oral Maxillofac Res 2018;9(3):e1

URL: http://www.ejomr.org/JOMR/archives/2018/3/e1/v9n3e1.pdf

doi: $10.5037 /$ jomr.2018.9301 


\section{INTRODUCTION}

Transverse mandibular discrepancy is characterized by unilateral or bilateral cross-bite, a tapered mandibular arch, crowded misaligned anterior teeth and a narrow inter-canine width [1-3]. Minor transverse mandibular discrepancies are generally corrected by orthodontic dental compensation, dental extraction, dental arch expansion or inter-approximal tooth stripping, whereas severe mandibular transverse discrepancies necessitates surgical intervention due to an early fusion of the symphysis and lack of a mandibular midsagittal suture $[\underline{1}, \underline{3}, \underline{4}]$. Transverse mandibular widening following a midline osteotomy and lateral rotation of the two hemi-mandible segments with an interpositional grafting material is a safe and predictable treatment modality for correction of moderate transverse mandibular discrepancies [5]. However, this surgical intervention is rarely used in patients with severe mandibular transverse discrepancies due to an increased risk of periodontal problems and relapse $[\underline{1}, \underline{2}, \underline{6}]$.

Mandibular midline distraction osteogenesis (MMDO) is a surgical technique to widen the mandible by incremental traction and gradually separating the mandibular symphysis in patients with severe mandibular transverse discrepancies $[\underline{1}, \underline{2}]$. MMDO was introduced by Rosenthal in 1951 [] and modified by Guerrero et al. [ㅁ] in the 1990s. MMDO can be achieved by the use of different types of distraction appliance (bone-borne, tooth-borne or hybrid distraction appliance) $[\underline{1}, \underline{2}, \underline{9-14}]$. Bone-borne distraction appliance has been recommended since the mechanical forces are delivered directly towards the bone facilitating a parallel basal mandibular bone widening with increased skeletal stability $[\underline{2}, \underline{10}, 11]$. However, bone-borne distraction appliance is associated with increased cost, extended duration of surgery, trans-mucosal hardware emergence and the need of a second operation to remove the distraction appliance $[\underline{1}, \underline{10}]$. Tooth-borne distraction appliance apply their vector on the dentoalveolar level and generally facilitate a disproportionate transverse expansion pattern with a larger alveolar bone widening than the basal mandibular bone as well as dental-tipping $[\underline{2}, \underline{12}, \underline{13}]$. However, toothborne distraction appliance is cheaper, cemented preoperatively and provides better aesthetic and patient comfort especially when a lingual device is used $[\underline{1}, \underline{12}, \underline{13}]$. Hybrid distraction appliance combines advantages of bone-borne and tooth-borne appliance, since the appliance is attached to both the bone and teeth $[\underline{13}, \underline{14}]$. Previous published biomechanical, experimental and human studies reveal transverse skeletal and dental arch expansion after MMDO with the different types of distraction appliance $[\underline{1}, \underline{2}, \underline{8}, \underline{15-}$ 19]. However, the transverse skeletal and dental arch expansion pattern and relapse following MMDO with a bone-borne, tooth-borne or hybrid distraction appliance have not yet been assessed specifically in a systematic review. Therefore, the objective of the present systematic review was to assess the transverse skeletal and dental arch expansion and relapse of the mandible after MMDO with a bone-borne, toothborne or hybrid distraction appliance.

\section{MATERIAL AND METHODS Protocol and registration}

The methods of the analysis and inclusion criteria were specified in advance and documented in a protocol. The review was registered in PROSPERO, an international prospective register of systematic reviews.

The protocol can be accessed at: https://www.crd. york.ac.uk/prospero/.

Registration number: CRD42018103295.

The present systematic review was conducted in accordance with the Preferred Reporting Items for Systematic reviews and Meta-Analyses (PRISMA) statement for reporting systematic reviews [20].

\section{Types of publications}

The present systematic review included studies on humans.

\section{Types of studies}

Randomized controlled trials, controlled trials, case series and retrospective studies.

\section{Types of outcome measures}

- Transverse skeletal expansion.

- Transverse skeletal relapse.

- Transverse dental arch expansion.

- Transverse dental arch relapse.

- Frequency of complications.

- Patient-reported outcome measures.

\section{Information sources}

The search strategy incorporated examinations of electronic databases, supplemented by a thorough hand-search page by page of relevant journals 
including "American Journal of Orthodontics", "American Journal of Orthodontics and Dentofacial Orthopedics", "British Journal of Oral and Maxillofacial Surgery", "European Journal of Orthodontics", "International Journal of Oral and Maxillofacial Surgery", "Journal of Craniofacial Surgery", "Journal of Cranio-Maxillo-Facial Surgery", "Journal of Oral \& Maxillofacial Research", "Journal of Oral and Maxillofacial Surgery", "Oral and Maxillofacial Surgery", "Oral Surgery Oral Medicine Oral Pathology Oral Radiology" and "The Angle Orthodontist". The manual search also included the bibliographies of all articles selected for full-text screening as well as previously published reviews relevant for the present systematic review. The search was performed by two of the authors (TSJ and TLB). Any disagreements were resolved by consensus between the two observers.

\section{Search}

A MEDLINE (PubMed), Embase, and Cochrane Library search was conducted. Human studies published in English until the $3^{\text {rd }}$ of July, 2018 were included. The search strategy was performed in collaboration with a librarian and utilized a combination of Medical subject heading (MeSH) and free text terms. The search strategy is outlined in Appendix 1 - 3.

\section{Selection of studies}

The titles of the identified reports were initially screened. The abstract was assessed when the title indicated that the study was relevant. Fulltext analysis was obtained for those with apparent relevance or when the abstract was unavailable. The references of the identified papers were crosschecked for unidentified articles. The search was performed by two of the authors (TSJ and TLB).
Any disagreements were resolved by consensus between the two observers.

\section{Study eligibility}

The inclusion criteria were developed using the PICOS guidelines (Table 1).

\section{Inclusion criteria}

The review exclusively focused on studies with an observation period of minimum three months after the end of the distraction period. The transverse skeletal and dental arch expansion or relapse after MMDO with bone-borne, tooth-borne or hybrid distraction appliance should be reported. In addition, at least five patients should be included in the study and the surgical technique, as well as the used distraction appliance must be clearly specified.

\section{Exclusion criteria}

Studies with insufficient description of the performed numbers of surgical procedures, significant dissimilarities in demographic data, lack of information on length of observation period and studies involving syndromic patients were excluded. Moreover, letters, editorials, $\mathrm{PhD}$ theses, letters to the editor, case reports, abstracts, technical reports, conference proceedings, animal or in vitro studies and literature review papers were also excluded.

\section{Data extraction}

Data were extracted by one reviewer (TSJ) according to a data-collection form ensuring systematic recording of the outcome measures. In addition, relevant characteristics of the study were recorded. The corresponding author was contacted by e-mail in the absence of important information or uncertainties.

Table 1. PICOS guidelines

\begin{tabular}{l|l}
\hline Patient and population (P) & $\begin{array}{l}\text { Healthy non-syndromic patients with a transverse mandibular deficiency requiring mandibular midline } \\
\text { distraction osteogenesis. }\end{array}$ \\
\hline Intervention (I) & Mandibular midline distraction osteogenesis. \\
\hline $\begin{array}{l}\text { Comparator or control } \\
\text { group (C) }\end{array}$ & Bone-borne distraction appliance, tooth-borne distraction appliance or hybrid distraction appliance. \\
\hline Outcomes (O) & $\begin{array}{l}\text { Transverse skeletal expansion and relapse of the mandible, transverse mandibular dental arch expansion } \\
\text { and relapse, frequency of complications and patient-reported outcome measures. }\end{array}$ \\
\hline Study design (S) & Randomized controlled trials, controlled trials, case series, retrospective studies. \\
\hline Focused question & $\begin{array}{l}\text { Are there any differences in the transverse mandibular skeletal and dental arch expansion and relapse } \\
\text { after mandibular midline distraction osteogenesis with a bone-borne distraction appliance, a tooth-borne } \\
\text { distraction appliance or a hybrid distraction appliance? }\end{array}$ \\
\hline
\end{tabular}




\section{Data items}

The following items were collected from the included articles and arranged in the following fields: study, year of publication, patients, transverse deficiency, distraction appliance, evaluation methods, followup, transverse skeletal expansion, transverse skeletal relapse, dental arch expansion, dental arch relapse, frequency of complications and patient reported outcome measures.

\section{Assessment of methodological quality}

The quality assessment of the included studies was undertaken as part of the data extraction process. A methodological quality rating system was used and the classification of the risk of bias potential for each study was based on the following five criteria:

- Random selection in the population (yes/no).

- Definition of inclusion and exclusion criteria (yes/no).

- Report of losses to follow-up (yes/no).

- Validated measurements (yes/no).

- Statistical analysis (yes/no).

The studies were grouped according to:

- Low risk of bias (plausible bias unlikely to seriously alter the results) if all above-described quality criteria were met.
- Moderate risk of bias (plausible bias that weakens confidence in the results) when one of these criteria were not included.

- High risk of bias (plausible bias that seriously weakens confidence in the results) when two or more criteria were missing.

\section{Statistical analysis}

Meta-analyses were to be conducted only if there were studies of similar comparison, reporting identical outcome measures. However, the studies included revealed considerable variations in study design, i.e. different latency period, distraction rates, length of consolidation period and follow-up, type of outcome measures as well as dissimilar evaluation methods. Therefore, a well-defined meta-analysis was not applicable. Parametric data were expressed as mean and standard deviation (M [SD]). Statistical significance level was defined at $\mathrm{P}=0.05$.

\section{RESULTS}

Study selection

Article review and data extraction were performed according to the PRISMA flow diagram (Figure 1).

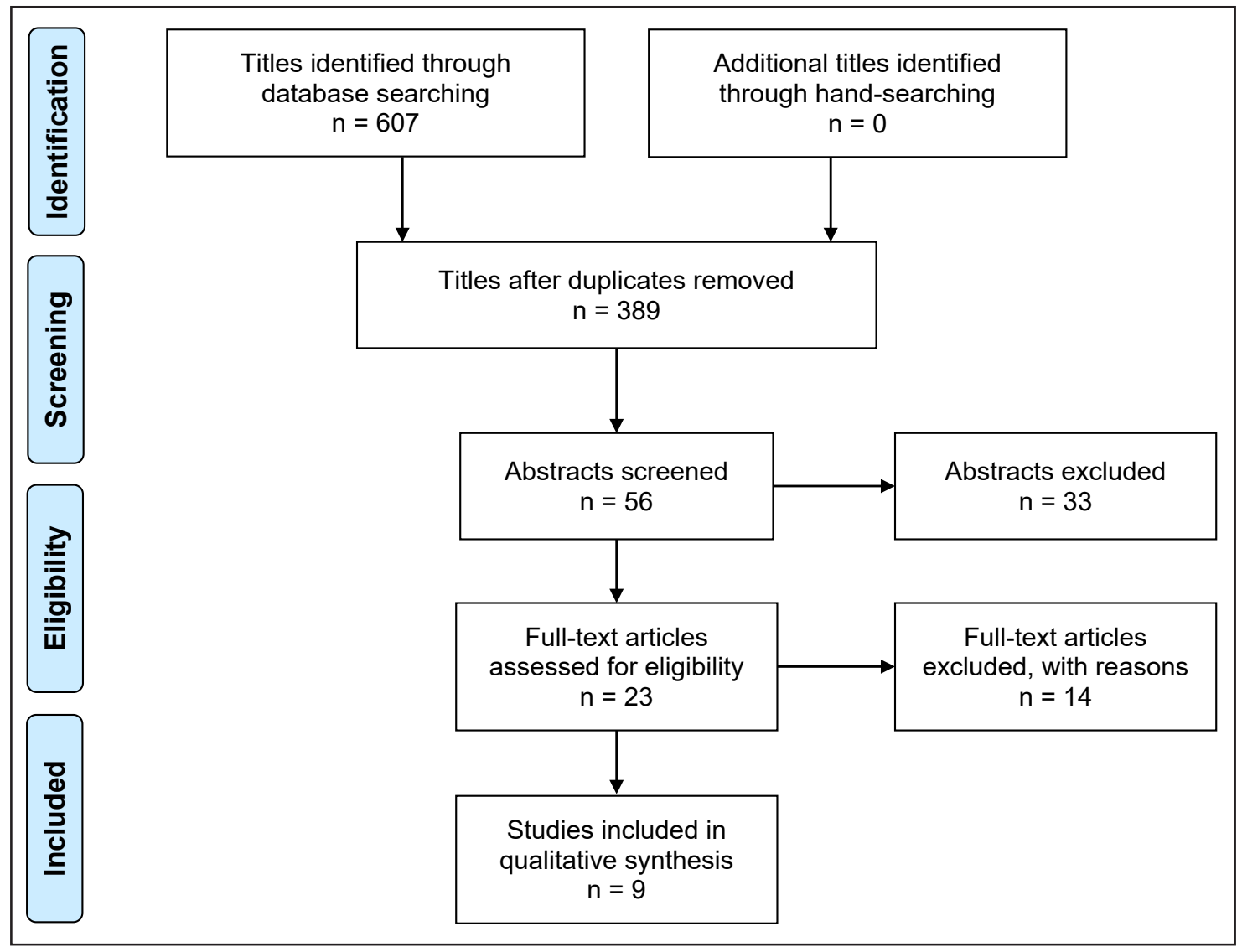

Figure 1. PRISMA flow diagram demonstrating the results of the systematic literature search. 
A total of 607 titles were identified and 56 abstracts were reviewed. Full-text analysis included 23 articles and nine studies were finally included in the present systematic review [2, 21-28]. No articles were included as the result of hand-searching.

\section{Exclusion of studies}

The reasons for excluding studies after full-text assessment were as follows: the transverse skeletal and dental arch expansion or relapse of the mandible after MMDD were not reported [10,29-31], toothborne appliance and hybrid appliance was used without differentiating the transverse skeletal and dental arch expansion or relapse between the two treatment modalities [32], the length of the observation period was not specified $[\underline{11}, \underline{13}, \underline{33}, \underline{34}]$. Finally, five studies $[14, \underline{35-38}]$ were excluded because the same patient sample was reported in other publications included in the present systematic review $[\underline{2}, \underline{28}]$.

\section{Study characteristics}

The included studies consisted of two comparative studies using a retrospective study design [2,21], four non-comparative case series $[\underline{22}, \underline{23}, \underline{26}, \underline{28}]$ and three non-comparative retrospective studies $[\underline{24}, \underline{25}, 27]$. Bone-borne (Surgi-Tec NV, Brugge, Belgium or Modus, Medartis ${ }^{\circledR}$, Basel, Switzerland), tooth-borne (Hyrax) and hybrid (Hyrax) appliance were compared in one study [2], while tooth-borne (Hyrax) and hybrid (Hyrax) appliance were compared in another study [21]. Non-comparative studies involved bone-borne (Modus by Medartis, SurgiTec and TMD-Flex Rotterdam) appliance in three studies [22-24], tooth-borne (Hyrax, custom-made or not specified) appliance in three studies [25-27], and hybrid (custom-made) appliance in one study [28]. A rigid intraoral distraction appliances were used in all the included studies [2, 21-28], apart from one study, in which some patients were treated with the flexible TMD-Flex Rotterdam distraction appliance [24]. No estimate of sample size or power calculation were conducted in any of the included studies. Moreover, detailed information about the transverse mandibular discrepancy, surgical intervention, retention period, blind assessment, assessor training, pre-distraction orthodontic expansion or relapse was infrequently specified. The number and skills of the surgeons involved in the surgical procedure were described in four studies $[\underline{2}, \underline{21}, \underline{22}, \underline{25}]$. The transverse mandibular deficit was reported in three studies [2, 22, 27]]. MMDO was performed in local anaesthesia [2, 23] intravenous sedation $[25,26,28]$ or general anaesthesia [22]. An active screw mechanism were used in all the included studies [2, 21-28]. The distraction appliance was activated after five days [2,르], five to seven days [2] $]$, seven days $[\underline{23}, \underline{27}, \underline{28}]$ and eight days [25]. Distraction rate was $0.4 \mathrm{~mm}$ per day [27], $0.6 \mathrm{~mm}$ per day $[22,26]$, and $1 \mathrm{~mm}$ per day $[2,23,25,28]$. Consolidation period was four weeks [23], six weeks [마], and three months $[\underline{2}, \underline{22}, \underline{25}, \underline{27}, \underline{28}]$. Frontal and lateral cephalograms involving dental measurements, ramal angle, bigonial and biantegonial distance was used to estimate the transverse skeletal expansion $[21,23,25,28]$, dental arch expansion [25] as well as skeletal relapse $[21,24,25,28]$. Dental cast measurements involving measurements at tooth and bone level was used to estimate the transverse skeletal expansion [26], dental arch expansion [21,23$\underline{26}, \underline{28}]$ as well as dental relapse $[21,24, \underline{28}]$. Computed tomography (CT) scan was used to estimate the transverse skeletal expansion [27] and dental arch expansion $[\underline{22}, 27]$. Frequency of complications was reported in five studies $[\underline{2}, \underline{22}, \underline{23}, \underline{26}, \underline{27}]$. Patientreported outcome measures were not reported in any of the included studies.

\section{Outcome measures}

The result of each outcome measure is presented first and then a short summary is finally provided. The results of the outcome measures are outlined in Table 2 - 6.

\section{Transverse skeletal expansion Comparative studies}

The transverse skeletal expansion was $4.6(0.9)$ $\mathrm{mm}$ with bone-borne appliance, 3.7 (1.1) $\mathrm{mm}$ with tooth-borne appliance and $4.6(0.9) \mathrm{mm}$ with hybrid appliance (Table 2) [2]. No statistically analysis was conducted and the method used for evaluating the transverse skeletal expansion was not described [2].

The immediate post-distraction transverse skeletal expansion between bone markers placed on either side of the symphysis was 2.3 (1.3) mm with a toothborne appliance compared to $5.3(1.4) \mathrm{mm}$ with a hybrid appliance, as evaluated by linear measurements on posterior-anterior cephalograms [21]. The interincisor apices width increased by $2.8(2.1) \mathrm{mm}$ with a tooth-borne appliance compared to $5.1(1.7) \mathrm{mm}$ with a hybrid appliance. Bigonial and biantegonial width decreased by $-1.9(6.2) \mathrm{mm}$ and $-1.3(5.1) \mathrm{mm}$ with a tooth-borne appliance compared to $-0.4(6.3) \mathrm{mm}$ and $0.7(4.4) \mathrm{mm}$ with a hybrid appliance. Statistical analysis was not conducted [21]. 
Table 2. Comparative studies assessing mandibular midline distraction osteogenesis with a bone-borne, tooth-borne or hybrid distraction appliance

\begin{tabular}{|c|c|c|c|c|c|c|c|c|c|c|c|c|c|c|}
\hline \multirow{3}{*}{ Study } & \multirow{3}{*}{$\begin{array}{c}\text { Year of } \\
\text { publication }\end{array}$} & \multirow{3}{*}{$\begin{array}{c}\text { Number of } \\
\text { patients }\end{array}$} & \multicolumn{4}{|c|}{ Materials and methods } & \multicolumn{8}{|c|}{ Outcomemeasures } \\
\hline & & & \multirow{2}{*}{$\begin{array}{l}\text { TMD } \\
(\mathrm{mm})\end{array}$} & \multirow{2}{*}{$\begin{array}{c}\text { Distraction } \\
\text { appliance }\end{array}$} & \multirow{2}{*}{$\begin{array}{c}\text { Evaluation } \\
\text { methods }\end{array}$} & \multirow{2}{*}{ Follow-up } & \multicolumn{2}{|c|}{$\begin{array}{c}\text { Transverse skeleta } \\
\text { expansion }\end{array}$} & \multicolumn{2}{|c|}{$\begin{array}{c}\text { Transverse skeletal } \\
\text { relapse }\end{array}$} & \multicolumn{2}{|c|}{$\begin{array}{c}\text { Dental arch } \\
\text { expansion }\end{array}$} & \multicolumn{2}{|c|}{$\begin{array}{l}\text { Dental arch } \\
\text { relapse }\end{array}$} \\
\hline & & & & & & & \multicolumn{2}{|c|}{$\begin{array}{l}\text { Mean (SD), } \\
\text { mm }\end{array}$} & \multicolumn{2}{|c|}{$\begin{array}{l}\text { Mean (SD), } \\
\text { mm }\end{array}$} & \multicolumn{2}{|c|}{$\begin{array}{l}\text { Mean (SD), } \\
\quad \text { mm }\end{array}$} & \multicolumn{2}{|c|}{$\begin{array}{l}\text { Mean (SD), } \\
\text { mm }\end{array}$} \\
\hline \multirow{3}{*}{ Alkan et al. [2] } & \multirow{3}{*}{2007} & 5 & \multirow{3}{*}{$>7$} & Bone-borne & \multirow{3}{*}{$\begin{array}{c}\text { Clinical } \\
\text { X-ray }\end{array}$} & \multirow{3}{*}{$\begin{array}{c}1.8 \text { years } \\
\text { (9 months } \\
\text { to } \\
3 \text { years) }\end{array}$} & & $(0.9)$ & \multirow{3}{*}{\multicolumn{2}{|c|}{ NR }} & & $(0.8)$ & \multirow{3}{*}{\multicolumn{2}{|c|}{ NR }} \\
\hline & & 21 & & Tooth-borne & & & & (1.1) & & & & $(0.9)$ & & \\
\hline & & 14 & & Hybrid & & & \multicolumn{2}{|c|}{$4.6(0.9)$} & & & \multicolumn{2}{|c|}{$5(0.9)$} & & \\
\hline \multirow{11}{*}{ Durham et al. [21] } & \multirow{11}{*}{2017} & \multirow{6}{*}{14} & \multirow{11}{*}{ NR } & \multirow{6}{*}{ Tooth-borne } & \multirow{11}{*}{$\begin{array}{c}\text { Dental cast } \\
\text { X-ray }\end{array}$} & \multirow{6}{*}{5.1 years } & Region & PS - PD & PS - PT & PS - FU & Region & PS - PD & PS - PT & PS - FU \\
\hline & & & & & & & BG & $-1.9(6.2)$ & $-1.8(8.2)$ & $-2.4(7.7)$ & $\mathrm{ICD}$ & $5.1(1.6)$ & $2.6(1.5)$ & $1.3(0.9$ \\
\hline & & & & & & & BAG & $-1.3(5.1)$ & $-1.6(5.5)$ & $-1.8(5.2)$ & IPMD1 & $4.6(1.4)$ & $2.1(1.4)$ & $1.2(1.7$ \\
\hline & & & & & & & $\mathrm{BM}$ & $2.3(1.3)$ & $1.5(1.8)$ & $1.6(2)$ & IPMD2 & $3.8(1.3)$ & $2(1.4)$ & $1.5(1.8$ \\
\hline & & & & & & & & & & & IMD1 & $2.5(1.8)$ & $2.8(1.6)$ & $3(1.9)$ \\
\hline & & & & & & & IIA & $2.8(2.1)$ & $-4.6(1.3)$ & $-3.4(0.7)$ & IMD2 & $1.1(2.1)$ & $2.5(2.3)$ & $2.6(2.5$ \\
\hline & & & & & & & BG & $-0.4(6.3)$ & $-1(6.2)$ & $-1.3(7.3)$ & ICD & $5.6(2.1)$ & $0.9(1)$ & $0.2(1.3$ \\
\hline & & & & & & & BAG & $0.7(4.4)$ & $0.3(4.3)$ & $1(5.3)$ & IPMD1 & $5.3(1.8)$ & $1.3(0.9)$ & $0.9(1.6$ \\
\hline & & 19 & & Hybrid & & 6.1 years & $\mathrm{BM}$ & $5.3(1.4)$ & $4.7(1.5)$ & $5.1(1.9)$ & IPMD2 & $4.4(2.2)$ & $1.1(1.5)$ & $1(1.6)$ \\
\hline & & & & & & & & & & & IMD1 & $3.1(2.5)$ & $1.9(1.8)$ & $2.5(2.1$ \\
\hline & & & & & & & 11A & $5.1(1.7)$ & $-2.4(1.1)$ & $-1.9(1.1)$ & IMD2 & $1.8(2.4)$ & $1.6(1.5)$ & $2.2(1.3$ \\
\hline
\end{tabular}

$\mathrm{BAG}=$ biantegonial; $\mathrm{BG}=$ bigonial; $\mathrm{BM}=$ bone marker; $\mathrm{FU}=$ follow-up; $\mathrm{ICD}=$ inter-canine distance; IIA = inter-incisor apices; IMD1 = first inter-molar distance; IMD2 = second inter-molar distance; IPMD1 = first inter-premolar distance; IPMD2 = second inter-premolar distance; NR = not reported; PD = post-distraction; PS = post-surgical; PT = posttreatment; $\mathrm{SD}=$ standard deviation; TMD = transverse mandibular deficit. 
Table 3. Non-comparative studies assessing mandibular midline distraction osteogenesis with a bone-borne distraction appliance

\begin{tabular}{|c|c|c|c|c|c|c|c|c|c|c|c|c|c|c|c|}
\hline \multirow{3}{*}{ Study } & \multirow{3}{*}{$\begin{array}{c}\text { Year of } \\
\text { publication }\end{array}$} & \multirow{3}{*}{$\begin{array}{c}\text { Number of } \\
\text { patients }\end{array}$} & \multicolumn{3}{|c|}{ Materials and methods } & \multicolumn{10}{|c|}{ Outcome measures } \\
\hline & & & \multirow{2}{*}{$\begin{array}{l}\text { TMD } \\
(\mathbf{m m})\end{array}$} & \multirow{2}{*}{$\begin{array}{c}\text { Evaluation } \\
\text { methods }\end{array}$} & \multirow{2}{*}{ Follow-up } & \multicolumn{2}{|c|}{$\begin{array}{c}\text { Transverse skeletal } \\
\text { expansion }\end{array}$} & $\begin{array}{c}\text { Transverse skeletal } \\
\text { relapse }\end{array}$ & \multicolumn{4}{|c|}{ Dental arch expansion } & \multicolumn{3}{|c|}{ Dental arch relapse } \\
\hline & & & & & & \multicolumn{2}{|c|}{$\begin{array}{l}\text { Mean (SD), } \\
\quad \text { mm }\end{array}$} & $\begin{array}{l}\text { Mean (SD), } \\
\text { mm }\end{array}$ & \multicolumn{4}{|c|}{$\begin{array}{l}\text { Mean (SD), } \\
\text { mm }\end{array}$} & \multicolumn{3}{|c|}{$\begin{array}{l}\text { Mean (SD), } \\
\text { mm }\end{array}$} \\
\hline \multirow{2}{*}{ Landes et al. [22] } & \multirow{2}{*}{2008} & \multirow{2}{*}{9} & \multirow{2}{*}{$>4$} & \multirow{2}{*}{ CT-scan } & \multirow{2}{*}{3 months } & \multirow{2}{*}{\multicolumn{2}{|c|}{ NR }} & \multirow{2}{*}{ NR } & Region & \multicolumn{3}{|c|}{$\mathrm{PO}-\mathrm{PD}$} & \multirow{2}{*}{\multicolumn{3}{|c|}{ NR }} \\
\hline & & & & & & & & & ICD & \multicolumn{3}{|c|}{$3.8(0.2)^{\mathrm{a}}$} & & & \\
\hline \multirow{7}{*}{ Gunbay et al. [23] } & \multirow{7}{*}{2009} & \multirow{7}{*}{7} & \multirow{7}{*}{ NR } & \multirow{7}{*}{$\begin{array}{c}\text { Dental cast } \\
\text { X-ray }\end{array}$} & \multirow{7}{*}{$\begin{array}{c}40 \text { months } \\
(36-48)\end{array}$} & Region & PO - PD & \multirow{7}{*}{ NR } & Region & & $\mathrm{PO}-\mathrm{PD}$ & & \multirow{7}{*}{\multicolumn{3}{|c|}{ NR }} \\
\hline & & & & & & & & & CID & & $2.1(1.8)^{\mathrm{b}}$ & & & & \\
\hline & & & & & & ( & $2.7(0.0)$ & & ICD & & $3.9(1.9)^{\mathrm{d}}$ & & & & \\
\hline & & & & & & $\mathrm{D} \wedge \mathrm{G}$ & $2(055 \mathrm{~b}$ & & IPMD1 & & $5.8(1.9)^{\mathrm{b}}$ & & & & \\
\hline & & & & & & & & & IPMD2 & & $5.1(1.5)^{b}$ & & & & \\
\hline & & & & & & & & & IMD1 & & $3.7(1)^{\mathrm{b}}$ & & & & \\
\hline & & & & & & & $-1.0(1.0)$ & & IMD2 & & $2.5(1.1)^{\mathrm{e}}$ & & & & \\
\hline & & & & & & Ramal a & ngle $^{\circ}$ & Ramal angle $^{\circ}$ & Region & $\mathrm{PO}-\mathrm{PD}$ & $\mathrm{PO}-1 \mathrm{Y}$ & $\mathrm{PO}-6.5 \mathrm{Y}$ & Region & $6.5 \mathrm{Y}-1 \mathrm{Y}$ & $1 \mathrm{Y}-\mathrm{PO}$ \\
\hline & & & & Dental cast & & PO - PD & $\mathrm{PO}-6.5 \mathrm{Y}$ & $1 Y-P D$ & ICD & $4.4(0.6)^{\mathrm{i}}$ & $2.9(0.5)^{\mathrm{i}}$ & $2(0.7)^{\mathrm{j}}$ & ICD & $-0.9(0.6)^{\mathrm{g}}$ & $-1.5(0.6)^{\mathrm{n}}$ \\
\hline 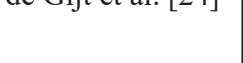 & 2010 & 17 & $1 \mathrm{NT}$ & X-ray & 0.0 yedis & $1{ }^{\prime}(07) f$ & & & IPMD & $4.9(0.8)^{\mathrm{i}}$ & $5.2(0.7)^{i}$ & $4.1(0.8)^{\mathrm{i}}$ & IPMD & $-1(0.7)^{1}$ & $0.3(0.7)^{\mathrm{g}}$ \\
\hline & & & & & & $-1.1(0.1)$ & $0(1.1)^{\circ}$ & $-1.7(0.1)$ & IMD & $2.4(0.4)^{\mathrm{i}}$ & $2.9(0.5)^{\mathrm{i}}$ & $3.8(0.8)^{\mathrm{k}}$ & IMD & $0.9(0.4)^{\mathrm{m}}$ & $0.4(0.3)^{\mathrm{g}}$ \\
\hline
\end{tabular}

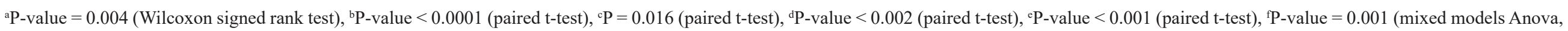

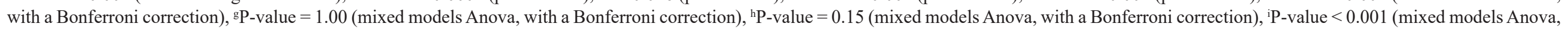

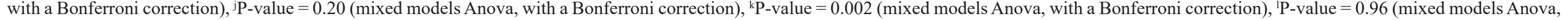
with a Bonferroni correction), ${ }^{\mathrm{m} P}$-value $=0.32$ (mixed models Anova, with a Bonferroni correction), ${ }^{\mathrm{n}} \mathrm{P}$-value $=0.16$ (mixed models Anova, with a Bonferroni correction).

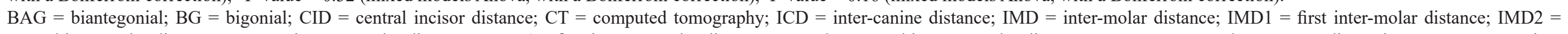

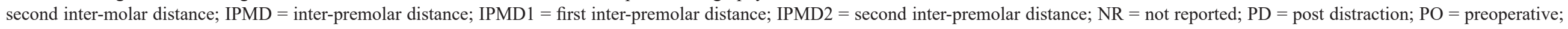
$\mathrm{SD}=$ standard deviation; $\mathrm{TMD}=$ transverse mandibular deficit; $\mathrm{Y}=$ year 
Table 4. Non-comparative studies assessing mandibular midline distraction osteogenesis with a tooth-borne distraction appliance

\begin{tabular}{|c|c|c|c|c|c|c|c|c|c|c|c|c|c|c|}
\hline \multirow{3}{*}{ Study } & \multirow{3}{*}{$\begin{array}{c}\text { Year of } \\
\text { publication }\end{array}$} & \multirow{3}{*}{$\begin{array}{c}\text { Number of } \\
\text { patients }\end{array}$} & \multicolumn{3}{|c|}{ Materials and methods } & \multicolumn{9}{|c|}{ Outcome measures } \\
\hline & & & \multirow{2}{*}{$\begin{array}{l}\text { TMD } \\
(\mathrm{mm})\end{array}$} & \multirow{2}{*}{$\begin{array}{l}\text { Evaluation } \\
\text { methods }\end{array}$} & \multirow[t]{2}{*}{ Follow-up } & \multicolumn{2}{|c|}{$\begin{array}{c}\text { Transverse skeletal } \\
\text { expansion }\end{array}$} & \multicolumn{2}{|c|}{$\begin{array}{c}\text { Transverse skeletal } \\
\text { relapse }\end{array}$} & \multicolumn{4}{|c|}{$\begin{array}{c}\text { Dental arch } \\
\text { expansion }\end{array}$} & \multirow{2}{*}{$\begin{array}{c}\begin{array}{c}\text { Dental arch } \\
\text { relapse }\end{array} \\
\text { Mean (SD), mm }\end{array}$} \\
\hline & & & & & & Mean & SD), mm & Mean & (SD), mm & & Mean (S & SD), $\mathbf{m m}$ & & \\
\hline \multirow{6}{*}{ Del Santo et al. [25] } & \multirow{6}{*}{2000} & \multirow{6}{*}{20} & \multirow{6}{*}{ NR } & \multirow{6}{*}{$\begin{array}{c}\text { Dental cast } \\
\mathrm{X} \text {-ray }\end{array}$} & \multirow{6}{*}{$\begin{array}{l}15 \text { months } \\
(6-31)\end{array}$} & Region & PO - PD & Region & PD - FFU & Region & $\mathrm{PO}-\mathrm{PD}$ & Region & PO - FFU & \multirow{6}{*}{ NR } \\
\hline & & & & & & BG & $0.7(4.4)$ & BG & $0.7(4.7)$ & \multirow{3}{*}{ ICD } & \multirow{3}{*}{$3.2(3.3)^{\mathrm{a}}$} & ICD & $2.4(1.9)^{\mathrm{b}}$ & \\
\hline & & & & & & BAG & $1.2(4.4)$ & BAG & $1.2(4.1)$ & & & IPMD1 & $3.5(2.4)^{b}$ & \\
\hline & & & & & & ICD & $3.2(3.3)^{\mathrm{a}}$ & $\mathrm{ICD}$ & $0.5(3.3)$ & & & IPMD2 & $4.9(3.9)^{\mathrm{b}}$ & \\
\hline & & & & & & \multirow{2}{*}{ IMD } & \multirow{2}{*}{$2.2(4.2)^{\mathrm{a}}$} & IMD & $-0.4(3)$ & \multirow{2}{*}{ IMD } & \multirow{2}{*}{$2.2(4.2)^{\mathrm{a}}$} & IMD1 & $5(3.2)^{b}$ & \\
\hline & & & & & & & & $\mathrm{BM}$ & $0.5(1.4)$ & & & IMD2 & $4.1(3.7)^{\mathrm{a}}$ & \\
\hline \multirow{6}{*}{ Ploder et al. [26] } & \multirow{6}{*}{2009} & \multirow{6}{*}{20} & \multirow{6}{*}{ NR } & \multirow{6}{*}{$\begin{array}{c}\text { Dental cast } \\
\text { X-ray }\end{array}$} & \multirow{6}{*}{3 months } & Region & PO - PD & \multirow{6}{*}{\multicolumn{2}{|c|}{ NR }} & Region & & PO - PD & & \\
\hline & & & & & & ICD & $3.4(2)$ & & & ICD & & $4.2(1.8)$ & & \\
\hline & & & & & & IPMD1 & $3.6(1.8)$ & & & IPMD1 & & $5(2)^{\mathrm{c}}$ & & \\
\hline & & & & & & IPMD2 & $3.3(1.4)$ & & & IPMD2 & & $4.7(2)^{\mathrm{c}}$ & & NR \\
\hline & & & & & & IMD1 & $3.2(1.5)$ & & & IMD1 & & $4.3(1.7)^{\mathrm{c}}$ & & \\
\hline & & & & & & IMD2 & $2.2(1.8)$ & & & IMD2 & & $3.6(1.3)$ & & \\
\hline & & & & & & Region & PO - PD & & & Region & & $\mathrm{PO}-\mathrm{PD}$ & & \\
\hline Seeheroer et al $[27$ & 2011 & 19 & $>4$ & CT-scan & 3 months & IPRD & $2.9(1.8)^{\mathrm{d}}$ & & $N R$ & IPD & & $4.8(1.6)^{\mathrm{d}}$ & & NR \\
\hline 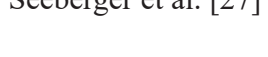 & 2011 & 19 & & Ci-scant & S & IMRP & $2.6(2.1)^{\mathrm{d}}$ & & 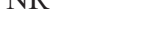 & IMD & & $4.9(1.3)^{\mathrm{d}}$ & & $\mathrm{N} \Lambda$ \\
\hline & & & & & & IMFD & $2.7(1.2)^{\mathrm{d}}$ & & & & & & & \\
\hline
\end{tabular}

${ }^{a} \mathrm{P}$-value $<0.05$ (paired t-test), ${ }^{\text {PP-value }}<0.01$ (paired t-test), ${ }^{\mathrm{CP}}$-value $<0.05$ (statistical test as not reported), ${ }^{\mathrm{d} P}$-value $<0.05$ (Wilcoxon signed rank test).

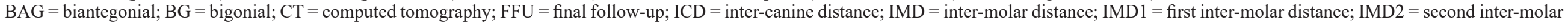

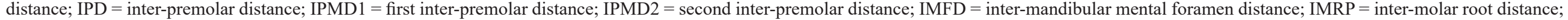
$\mathrm{IPRD}=$ inter-premolar root distance; $\mathrm{PD}=$ post-distraction; $\mathrm{PO}=$ preoperative; $\mathrm{NR}=$ not reported; $\mathrm{SD}=$ standard deviation; $\mathrm{TMD}=$ transverse mandibular deficiency.

Table 5. Non-comparative studies assessing mandibular midline distraction osteogenesis with a hybrid distraction appliance

\begin{tabular}{|c|c|c|c|c|c|c|c|c|c|c|c|c|c|c|c|}
\hline \multirow{3}{*}{ Study } & \multirow{3}{*}{$\begin{array}{c}\text { Year of } \\
\text { publication }\end{array}$} & \multirow{3}{*}{$\begin{array}{l}\text { Number of } \\
\text { patients }\end{array}$} & \multicolumn{3}{|c|}{ Materials and methods } & \multicolumn{10}{|c|}{ Outcome measures } \\
\hline & & & \multirow{2}{*}{$\begin{array}{l}\text { TMD } \\
(\mathbf{m m})\end{array}$} & \multirow{2}{*}{$\begin{array}{l}\text { Evaluation } \\
\text { methods }\end{array}$} & \multirow[t]{2}{*}{ Follow-up } & \multicolumn{3}{|c|}{$\begin{array}{c}\text { Transverse skeletal } \\
\text { expansion }\end{array}$} & \multirow{2}{*}{\multicolumn{2}{|c|}{$\begin{array}{c}\text { Transverse skeletal } \\
\text { relapse } \\
\text { Mean (SD), mm } \\
\end{array}$}} & \multirow{2}{*}{\multicolumn{3}{|c|}{$\begin{array}{c}\begin{array}{c}\text { Dental arch } \\
\text { expansion }\end{array} \\
\text { Mean (SD), mm } \\
\end{array}$}} & \multirow{2}{*}{\multicolumn{2}{|c|}{$\begin{array}{c}\text { Dental arch } \\
\text { relapse } \\
\text { Mean (SD) mm }\end{array}$}} \\
\hline & & & & & & Mea & $\mathrm{n}(\mathrm{SD}), \mathrm{mn}$ & & & & & & & & \\
\hline \multirow{6}{*}{ Malkoc et al. [28] } & \multirow{6}{*}{2006} & \multirow{6}{*}{20} & \multirow{6}{*}{ NR } & \multirow{6}{*}{$\begin{array}{l}\text { Dental cast } \\
\text { X-ray }\end{array}$} & \multirow{6}{*}{$\begin{array}{l}24.1(4.2) \\
\text { months }\end{array}$} & Region & \begin{tabular}{|l|}
$\mathrm{PO}-\mathrm{PD}$ \\
\end{tabular} & $\mathrm{PO}-\mathrm{FFU}$ & \begin{tabular}{l|l} 
Region \\
\end{tabular} & PD - FFU & Region & $\mathrm{PO}-\mathrm{PD}$ & PO - FFU & Region & PD - FFU \\
\hline & & & & & & \multirow{3}{*}{ BG } & \multirow{3}{*}{$-0.3(6.3)$} & \multirow{3}{*}{$-0.2(6.2)$} & \multirow{3}{*}{ BG } & \multirow{3}{*}{$0.1(6.2)$} & ICD & $7.3(2.1)^{\mathrm{a}}$ & $4.8(1.5)^{\mathrm{a}}$ & ICD & $-2.5(1.5)^{\mathrm{a}}$ \\
\hline & & & & & & & & & & & IPMD1 & $6.7(2.7)^{\mathrm{a}}$ & $5.5(2.2)^{\mathrm{a}}$ & IPMD1 & $-1.2(2.2)^{b}$ \\
\hline & & & & & & & & & & & IPMD2 & $4.8(3.1)^{\mathrm{a}}$ & $4.8(3)^{\mathrm{a}}$ & IPMD2 & $0(3)$ \\
\hline & & & & & & \multirow{2}{*}{ Ramal angle $^{\circ}$} & \multirow{2}{*}{$-0.1(5.9)$} & \multirow{2}{*}{$-0.1(5.3)$} & \multirow{2}{*}{ Ramal angle $^{\circ}$} & \multirow{2}{*}{$0(5.3)$} & IMD1 & $3.3(3.4)^{\mathrm{a}}$ & $3.7(3.3)^{\mathrm{a}}$ & IMD1 & $0.4(3.3)$ \\
\hline & & & & & & & & & & & IMD2 & $1.4(3)^{\mathrm{a}}$ & $1.9(3.4)^{\mathrm{a}}$ & IMD2 & $0.5(3.4)$ \\
\hline
\end{tabular}

${ }^{a}$ P-value $<0.001$ (paired t-test), ${ }^{b}$ P-value $<0.01$ (paired t-test).

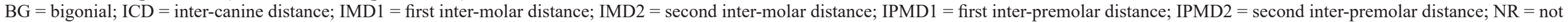
reported, $\mathrm{PD}=$ post-distraction; $\mathrm{PO}=$ preoperative; $\mathrm{PR}=$ post-retention; $\mathrm{PT}=$ post-treatment $\mathrm{SD}=$ standard deviation; $\mathrm{TMD}=$ transverse mandibular deficiency 
Table 6. Complications after mandibular midline distraction osteogenesis with a bone-borne, tooth-borne or hybrid distraction appliance

\begin{tabular}{c|c|l}
\hline \multicolumn{1}{c|}{ Study } & Distraction appliance & \multicolumn{1}{c}{ Type of complication } \\
\hline \multirow{2}{*}{ Alkan et al. [2] } & Bone-borne & $\begin{array}{l}\text { Breakage distractor: } 3 \\
\text { Ecchymosis: } 1 \\
\text { Gingival recession with excessive mobility of central incisors: } 1 \\
\text { Secondary infection: } 1 \\
\text { Chin ptosis: } 1\end{array}$ \\
\cline { 2 - 3 } & Tooth-borne & $\begin{array}{l}\text { Severe mucosal irritation: } 1 \\
\text { Failure: } 1\end{array}$ \\
\cline { 2 - 3 } & Hybrid & $\begin{array}{l}\text { Ecchymosis: } 1 \\
\text { Secondary infection: } 1\end{array}$ \\
\hline Landes et al. [22] & Bone-borne & None \\
\hline \multirow{2}{*}{ Gunbay et al. [23] } & Bone-borne & $\begin{array}{l}\text { Central incisor damage: } 1 \\
\text { Wound dehiscence: } 3 \\
\text { Temporomandibular joint pain: } 3 \\
\text { Gingivitis: } 7\end{array}$ \\
\hline \multirow{2}{*}{ Ploder et al. [26] } & Tooth-borne & $\begin{array}{l}\text { Temporomandibular joint pain: } 1 \\
\text { Temporomandibular joint click: } 1 \\
\text { Central incisor late response to cold testing: } 4\end{array}$ \\
\hline Seeberger et al. [27] & Tooth-borne & None \\
\hline
\end{tabular}

\section{Non-comparative studies}

The immediate post-distraction distance between the bigonial and biantegonial significantly increased by $2.4(0.5) \mathrm{mm}(\mathrm{P}<0.0001)$ and $2.3(0.5) \mathrm{mm}(\mathrm{P}<$ $0.0001)$ with a bone-borne appliance compared to preoperative measurements, as evaluated by linear measurements on posterior-anterior cephalograms (Table 3) [23].

The immediate post-distraction ramal angle significantly decreased by $-1.6(1.3)$ degrees $(\mathrm{P}=$ $0.016)[23]$ and $-1.1(0.7)$ degrees $(P=0.001)$ with a bone-borne distraction appliance [24].

The immediate post-distraction distance between the bigonial and biantegonial non-significantly increased by $0.7(4.4) \mathrm{mm}$ and $1.2(4.4) \mathrm{mm}$ with a tooth-borne appliance, as evaluated by linear measurements on posterior-anterior cephalograms (Table 4) [25].

The 3-months transverse skeletal expansion at the bone level between the canines, second premolar and second molar was 3.4 (2) $\mathrm{mm}, 3.3$ (1.4) $\mathrm{mm}$ and $2.2(1.8) \mathrm{mm}$ with a tooth-borne appliance, as evaluated by linear measurements on dental casts [26]. Statistical analysis was not conducted [26].

The 3-months transverse skeletal expansion between the mental foramen and the roots of pre-molars and molars was significantly increased by $2.7(1.2) \mathrm{mm}$ $(\mathrm{P}<0.05), 2.9(1.8) \mathrm{mm}(\mathrm{P}<0.05)$, and $2.6(2.1) \mathrm{mm}$ $(\mathrm{P}<0.05)$ with a tooth-borne appliance, as evaluated by linear measurements on CT-scans [27].

The immediate post-distraction bigonial distance decreased non-significantly by $0.3(6.3) \mathrm{mm}$ with a hybrid appliance, as evaluated by linear measurements on posterior-anterior cephalograms (Table 5) [28].
The ramal angle decreased non-significantly by 0.1 (5.9) degrees [28].

Summary

Comparative studies demonstrated transverse skeletal expansion with the three treatment modalities. Hybrid appliance created more immediate post-distraction transverse skeletal expansion compared to tooth-borne appliance, as evaluated by symphyseal bone markers measurements and inter-incisor apical width, whereas the bigonial and biantegonial width decreased with tooth-borne appliance compared to hybrid appliance. Non-comparative studies demonstrated a significant increase in the bigonial and biantegonial distance with a bone-borne appliance, whereas non-significant differences were disclosed with tooth-borne and hybrid appliance.

\section{Transverse skeletal relapse Comparative studies}

The transverse skeletal relapse was estimated by comparing the immediate post-distraction measurements to measurements obtained after the end of the active orthodontic treatment and follow-up examination (Table 2) [21]. Linear measurements on posterior-anterior cephalograms of symphysis bone markers revealed a relapse of $0.8(1.7) \mathrm{mm}$ at the end of the active orthodontic treatment and 0.7 (2) $\mathrm{mm}$ after 5.1 years with a tooth-borne appliance compared to $0.6(1.5) \mathrm{mm}$ and 0.2 (1.9) $\mathrm{mm}$ with a hybrid appliance, after 6.1 years. The inter-incisor apices distance decreased by $7.4(1.3) \mathrm{mm}$ and $6.2(0.7) \mathrm{mm}$ 
with a tooth-borne appliance compared to 7.5 (1.1) $\mathrm{mm}$ and 7 (1.1) $\mathrm{mm}$ with a hybrid appliance. The bigonial width increased by 0.1 (8.2) $\mathrm{mm}$ and decreased by $0.5(7.7) \mathrm{mm}$ with a tooth-borne appliance compared to a decrease of 0.6 (6.2) $\mathrm{mm}$ and 0.9 (7.3) $\mathrm{mm}$ with a hybrid appliance. The biantegonial width decreased by $0.3(5.5) \mathrm{mm}$ and 0.5 (5.2) $\mathrm{mm}$ with a tooth-borne appliance compared to a decrease of 0.4 (4.3) $\mathrm{mm}$ and an increase of 0.3 (5.3) $\mathrm{mm}$ with a hybrid appliance. Statistical analysis was not conducted [21].

\section{Non-comparative studies}

The ramal angle decreased non-significantly by -1.7 (0.7) degrees $(\mathrm{P}=0.15)$ after one year compared to the immediate post-distraction measurements with a bone-borne appliance (Table 3) [24]. The ramal angle was non-significantly changed by 0 (1.1) degrees $(\mathrm{P}=1)$ after 6.5 years compared to preoperative measurements [24].

The bigonial and biantegonial width increased by 0.7 (4.7) $\mathrm{mm}$ and $1.2(4.1) \mathrm{mm}$ after 1.3 years compared to immediate post-distraction measurements with a tooth-borne appliance, as evaluated by linear measurements on posterior-anterior cephalograms (Table 4) [25]. The inter-molar distance decreased with 0.4 (3) $\mathrm{mm}$, whereas the inter-canine distance increased with $0.5(3.3) \mathrm{mm}$. Statistical analysis was not conducted [25].

The bigonial width increased non-significant by 0.1 (6.2) $\mathrm{mm}$ after 24.1 months compared to immediate post-distraction measurements with a hybrid appliance, as evaluated by linear measurements on posterioranterior cephalograms [28]. The ramal angle difference was non-significantly changed by 0 (5.3) degrees (Table 5) [28].

\section{Summary}

Comparative studies revealed a similar long-term transverse skeletal relapse pattern with tooth-borne and hybrid appliance. Non-comparative studies disclosed non-significant differences in the transverse skeletal relapse with bone-borne and hybrid appliance compared to preoperative and immediate postdistraction measurements.

\section{Transverse dental expansion Comparative studies}

The transverse dental expansion was $5 \mathrm{~mm}(0.8)$ with a bone-borne appliance, $4.9 \mathrm{~mm}(0.9)$ with a tooth-borne appliance, and $5(0.9) \mathrm{mm}$ with a hybrid appliance (Table 2) [2]. The method used for measurement of the transverse dental expansion was not described and statistical analysis was not conducted [2]

The immediate post-distraction transverse dental expansion between the canines, second premolars and second molars was $5.1(1.6) \mathrm{mm}, 3.8(1.3) \mathrm{mm}$, and $1.1(2.1) \mathrm{mm}$ with a tooth-borne appliance compared to $5.6(2.1) \mathrm{mm}, 4.4(2.2) \mathrm{mm}$, and $1.8(2.4) \mathrm{mm}$ with a hybrid appliance, as evaluated by linear dental cast measurements [21]. Statistical analysis was not conducted [21].

\section{Non-comparative studies}

The 3-months transverse dental expansion between the canines was $3.8(1.8) \mathrm{mm}(\mathrm{P}=0.004)$ compared to preoperative measurements with a bone-borne appliance, as evaluated by linear measurements on CT-scans (Table 3) [22].

The immediate post-distraction transverse dental expansion between the canines, second premolars and second molars was $3.9(1.9) \mathrm{mm}(\mathrm{P}<0.002), 5.1$ (1.5) $\mathrm{mm}(\mathrm{P}<0.0001)$ and $2.5(1.1) \mathrm{mm}(\mathrm{P}<0.001)$ compared to preoperative measurements with a boneborne appliance, as evaluated by linear dental cast measurements [23].

The immediate post-distraction transverse dental expansion between the canines, first premolars and first molars was $4.4(0.6) \mathrm{mm}(\mathrm{P}<0.001), 4.9$ $(0.8) \mathrm{mm}(\mathrm{P}<0.001)$ and $2.4(0.4) \mathrm{mm}(\mathrm{P}<0.001)$ compared to preoperative measurements with a boneborne appliance, as evaluated by linear dental cast measurements [24].

The immediate post-distraction transverse dental expansion between the canines and molars was 3.2 (3.3) $\mathrm{mm}(\mathrm{P}<0.05)$ and $2.2(4.2) \mathrm{mm}(\mathrm{P}<0.05)$ compared to preoperative measurements, with a toothborne appliance, as evaluated by linear measurements on posterior-anterior cephalograms (Table 4) [25]. The 1.3-year transverse dental expansion between the canines, second premolar and second molar was $2.4(1.9) \mathrm{mm}(\mathrm{P}<0.01), 4.9(3.9) \mathrm{mm}(\mathrm{P}<0.01)$ and 4.1 (3.7) $\mathrm{mm}(\mathrm{P}<0.05)$ compared to preoperative measurements with a tooth-borne appliance, as evaluated by linear dental cast measurements [25].

The 3-months transverse dental expansion between the canines, second premolars and second molars was $4.2(1.8) \mathrm{mm}, 4.7$ (2) $\mathrm{mm}$ and $3.6(1.3) \mathrm{mm}$ compared to preoperative measurements with a toothborne appliance, as evaluated by linear dental cast measurements Statistical analysis was not conducted [26]. 
The 3-months transverse dental expansion between the premolars and molars was $4.8(1.6) \mathrm{mm}(\mathrm{P}<0.05)$ and $4.9(1.3) \mathrm{mm}(\mathrm{P}<0.05)$ compared to preoperative measurements with a tooth-borne appliance, as evaluated by linear measurements on CT-scans [27].

The immediate post-distraction transverse dental expansion between the canines, second premolars and second molars was $7.3(2.1) \mathrm{mm}(\mathrm{P}<0.001)$, 4.8 (3.1) $\mathrm{mm}(\mathrm{P}<0.001)$ and $1.4(1.9) \mathrm{mm}(\mathrm{P}<$ $0.001)$ compared to preoperative measurements with a hybrid appliance, as evaluated by linear dental cast measurements (Table 5) [료]. Corresponding measurements were 4.8 (1.5) $\mathrm{mm}(\mathrm{P}<0.001), 4.8$ (3) $\mathrm{mm}(\mathrm{P}<0.001)$ and $1.9(3.4) \mathrm{mm}(\mathrm{P}<0.05)$, after 24.1-months [28 ].

\section{Summary}

Comparative studies revealed transverse dental expansion with the three treatment modalities. Noncomparative studies demonstrated a statistically significant immediate post-distraction transverse dental expansion with the three treatment modalities compared to preoperative measurements.

\section{Transverse dental relapse Comparative studies}

Transverse dental relapse was estimated by comparing post-distraction linear dental cast measurements to measurements obtained after the end of the active orthodontic treatment and the follow-up examination (Table 2) [21]. The relapse at the end of orthodontic treatment between the canines, second premolars and second molars was 2.5 (1.5) mm, 1.8 (1.4) $\mathrm{mm}$ and $-1.4(2.3) \mathrm{mm}$ with a tooth-borne appliance compared to 4.7 (1) $\mathrm{mm}, 3.3(1.5) \mathrm{mm}$, and 0.2 (1.5) $\mathrm{mm}$ with a hybrid appliance. The relapse at the follow-up examination between the canines, second premolars and second molars was $3.8(0.9) \mathrm{mm}, 2.2(1.8) \mathrm{mm}$, and $-1.4(2.5) \mathrm{mm}$ with a tooth-borne appliance compared to $5.4(1.3) \mathrm{mm}, 3.4$ (1.6) $\mathrm{mm}$, and -0.3 (1.3) $\mathrm{mm}$ with a hybrid appliance. Statistical analysis was not conducted [21].

\section{Non-comparative studies}

The width distance between the canines, premolars and molars was non-significantly changed by $-0.9(0.6) \mathrm{mm}(\mathrm{P}=1),-1(0.7) \mathrm{mm}$ $(\mathrm{P}=0.96)$ and $0.9(0.4) \mathrm{mm}(\mathrm{P}=0.32)$ after 6.5 years compared to measurements after one-year with a bone-borne appliance, as evaluated by linear dental cast measurements (Table 3) [24].
Corresponding measurements was $-1.5(0.6) \mathrm{mm}(\mathrm{P}=$ $0.16), 0.3(0.7) \mathrm{mm}(\mathrm{P}=1)$ and $0.4(0.3) \mathrm{mm}(\mathrm{P}=1)$ after one-year compared to immediate post-distraction measurements [24].

The width distance was significantly changed by $-2.5(1.5) \mathrm{mm}(\mathrm{P}<0.001)$ at the canines and -1.2 (2.2) $\mathrm{mm}(\mathrm{P}<0.01)$ at the first pre-molars and nonsignificantly changed by $0(3) \mathrm{mm}$ at the second premolar, $0.4(3.3) \mathrm{mm}$ at the first molars, and 0.5 (3.4) at the second molars after 24.1 months compared to immediate post-distraction measurements with a hybrid appliance, as evaluated by linear dental cast measurements (Table 5) [2ㅇ] .

\section{Summary}

Postsurgical orthodontic alignment is often initiated, three to six months after MMDO and the transverse dental relapse is commonly evaluated by linear dental cast measurements. Consequently, the definite long-term transverse dental relapse after MMDO is influenced by the postsurgical orthodontic treatment and is challenging to estimate. The transverse dental relapse was reported in a comparative study disclosing increased transverse dental relapse with a hybrid appliance compared to a tooth-borne appliance. A non-comparative study disclosed a non-significant long-term difference in the transverse dental relapse compared to measurements after one-year with a bone-borne appliance.

\section{Frequency of complications Comparative studies}

The frequency of complications was higher with bone-borne appliance compared to tooth-borne or hybrid appliance (Table 6) temporomandibular joint pain have been reported with [2] . Breakage of distractor, ecchymosis and secondary infection were the most commonly observed complications after MMDO with bone-borne appliance [2]

\section{Non-comparative studies}

Damage to the central incisor during the vertical osteotomy, gingivitis, wound dehiscence and temporomandibular joint pain have been reported with bone-borne appliance (Table 6) [23]. Delayed response to cold testing of the central incisor, temporomandibular joint pain and click has been reported with tooth-borne appliance [26], whereas no complications has been reported in non-comparative studies with the use of hybrid appliance. 


\section{Summary}

The frequency of complications was higher with bone-borne appliance compared to tooth-borne and hybrid appliance in comparative and non-comparative studies.

\section{Quality assessment}

The quality of the included studies is summarized in Table 7. All the included studies were considered with a high risk of bias [2, 21-28].

\section{DISCUSSION}

The objective of the present systematic review was to assess the transverse skeletal and dental arch expansion and relapse of the mandible after MMDO with a bone-borne, tooth-borne or hybrid distraction appliance. Two comparative and seven non-comparative studies with high risk of bias fulfilled the inclusion criteria [2,21-28]. Transverse skeletal and dental arch expansion of the mandible was achieved with the three treatment modalities [2,21-28]. Bone-borne and hybrid distraction appliance seem to facilitate more skeletal expansion compared with tooth-borne appliance, whereas no difference in dental arch expansion was reported [2,21]. Frequency of complications was higher with bone-borne appliance compared with tooth-borne and hybrid appliance. However, no randomized controlled trials were included in the present systematic review. Moreover, different latency periods, distraction rates, distraction vector, length of consolidation period and follow-up, type of outcome measures, dissimilar evaluation methods as well as various methodological confounding factors posed serious restrictions to review the literature in a quantitative systematic manner. Hence, the conclusions drawn from the results of the present systematic review should be interpreted with caution and well-designed longterm randomized controlled trials applying threedimensional technology, an economic perspective as well as patient-related outcome measures with the three treatment modalities are needed before definite conclusions can be provided.

High quality randomized controlled trials with low risk of bias provide the highest level of evidence for ascertaining the safety and efficacy of a specific surgical intervention. Previous published studies assessing MMDO with bone-borne, tooth-borne or hybrid appliance involves solely non-randomized trials, case series, retrospective studies and several case reports $[\underline{1}, \underline{2}, 21-38]$. Consequently, the current level of evidence is inadequate to propose implication for evidence based clinical guidelines. Thus, the treatment of choice for MMDO with the different types of distraction appliance should be case specific, less invasive and achieve the optimal treatment goal in the shortest period of time with less risk of biological and technical complications.

Dental and skeletal structural changes after MMDO with bone-borne, tooth-borne or hybrid distraction appliances have previously been assessed in a systematic review revealing a horizontal V-shaped opening with a larger anterior transverse mandibular expansion declining progressively towards the posterior part of the mandible $[\underline{1}, 2]$, which is in accordance with the results of the present systematic review. Moreover, a tooth-borne appliance seems to exhibit a vertical V-shaped widening of the mandible with larger transverse expansion at the dentoalveolar level compared to the basal bone level, whereas bone-borne and hybrid appliance facilitates a more symmetrical vertical widening $[1,2]$. However, previous published studies assessing the transverse mandibular expansion pattern after MMDO are largely based on dental cast measurements and two-dimensional radiographs [2, 21, 23-26, 28]

Table 7. Quality assessment of included studies

\begin{tabular}{l|c|c|c|c|c|c}
\hline \multicolumn{1}{c|}{ Study } & $\begin{array}{c}\text { Random selection } \\
\text { in the population }\end{array}$ & $\begin{array}{c}\text { Definition of inclusion } \\
\text { and exclusion criteria }\end{array}$ & $\begin{array}{c}\text { Report of losses } \\
\text { to follow-up }\end{array}$ & $\begin{array}{c}\text { Validated } \\
\text { measurements }\end{array}$ & $\begin{array}{c}\text { Statistical } \\
\text { analysis }\end{array}$ & $\begin{array}{c}\text { Risk of } \\
\text { bias }\end{array}$ \\
\hline Alkan et al. [2] & No & Yes & No & Yes & Yes & High \\
\hline Durham et al. [21] & No & Yes & No & Yes & Yes & High \\
\hline Landes et al. [22] & No & Yes & No & Yes & Yes & High \\
\hline Gunbay et al. [23] & No & Yes & No & Yes & Yes & High \\
\hline de Gijt et al. [24] & No & Yes & No & Yes & Yes & High \\
\hline Ploder et al. [26] & No & Yes & No & Yes & Hes & High \\
\hline Del Santo et al. [25] & No & Yes & No & Yes & Yes & High \\
\hline Seeberger et al. [27] & No & Yes & No & Yes & Yes & High \\
\hline Malkoc et al. [28] & No & Yes & &
\end{tabular}


These evaluation methods may be imprecise for measurement of the accurate basal skeletal expansion and relapse, due to superimposition of anatomic structures, difficulties to determine landmarks with high accuracy and postoperative orthodontic realignment of the teeth $[39, \underline{40}]$. Cone beam CT and CT-scan demonstrate a high degree of reproducibility including three-dimensional quantitation of bone changes and tooth inclination, which appears to be higher compared to frontal and lateral cephalometric radiographs [1ㅜ, $\underline{42}]$. The transverse mandibular expansion pattern after MMDO with a tooth-borne appliance has previously been assessed using CT disclosing a larger dental arch expansion compared to skeletal expansion [27]. Consequently, the transverse mandibular expansion pattern after MMDO with bone-borne, tooth-borne or hybrid appliance is dissimilar. Thus, the magnitude of the transverse mandibular deficiency and patient's preference must be taken into account, before selecting the type of distraction appliance for MMDO.

Patient-reported outcome measures are essentially reports of patients' perceptions of their oral health status and its impact on their daily life or quality of life. However, none of the included studies of the present systematic review assessed patientreported outcome measures after MMDO. A previous published study assessing patient's point of view, ease of use and overall patient satisfaction after surgical assisted rapid maxillary expansion with a boneborne distraction appliance compared with a toothborne appliance demonstrated an overall satisfaction rate over $90 \%$ for both distraction appliances [43]. However, bone-borne appliance was statistically significant easy to use compared with a tooth-borne appliance [43]. MMDO with a lingually placed tooth-borne appliance has been advocated, since it is minimally invasive and more comfortable for the patient [2]. Moreover, the use of a bone-borne and hybrid appliance increases the length of surgery and necessitates a second operation to remove the hardware [44]. Consequently, further studies assessing MMDO with a bone-borne, tooth-borne or hybrid appliance should include patient-reported outcome measures to establish benefits for the patients and provide information for an assessment of the optimal treatment goal in the shortest period of time with less risk of biological and technical complications.

The most commonly reported complications after MMDO with bone-borne, tooth-borne or hybrid appliance are wound dehiscence, pressure ulcer, distraction appliance-related problems, infection and tooth damage $[\underline{1}, \underline{2}, \underline{22}, \underline{23}, \underline{26}, \underline{27}, \underline{44}, \underline{45}]$. Intra- and postoperative complications were not reported in all the included studies of the present systematic review, but when reported, they were generally low and not serve $[\underline{2}, \underline{22}, \underline{23}, \underline{26}, \underline{27}]$. The frequency of complications was higher with bone-borne appliance compared with tooth-borne and hybrid appliance, which is in accordance with the results of previous published studies $[1,2,30,31]$. Excessive mobility of the central incisors was reported in one study after MMDO with a bone-borne appliance [2]. Tooth mobility and widening of the periodontal ligament adjacent to the osteotomy has previously been reported after MMDO $[45,46]$, but periodontal and dental morbidity after MMDO with a bone-borne appliance seem to be transient and limited to the distraction and consolidation period [46]. Intraoperative damage to the central incisors during the vertical osteotomy has previously been described in the literature and reported in one of the included studies of the present systematic review $[23,30]$. A mandibular midline step osteotomy has been suggested in patients with severe crowding to avoid intraoperative damage of the central incisors during the vertical osteotomy $[\underline{30}, \underline{47}]$. MMDO causes rotational movements of the mandibular condyles during the distraction phase [48]. Temporomandibular joint pain and click after MMDO have previously been described in the literature and reported in two of the included studies of the present systematic review $[\underline{23}, \underline{26}, \underline{30}]$. Permanent temporomandibular joint symptoms after MMDO are uncommon [23] and the temporomandibular pain in the included studies resolved with physiotherapy [23] and removal of the tooth-borne appliance [26].

\section{CONCLUSIONS}

The present systematic review demonstrates that mandibular midline distraction osteogenesis with a bone-borne, tooth-borne or hybrid distraction appliance is a safe and effective treatment modality to correct transverse mandibular discrepancies. Boneborne and hybrid appliance facilitate more skeletal expansion compared to tooth-borne appliance, whereas comparable dental arch expansion was observed with the different types of distraction appliance. Limited skeletal and dental arch relapse was observed with the different type of distraction appliances. Frequency of complications was higher with bone-borne appliance compared with toothborne or hybrid appliance. However, two comparative and seven non-comparative studies with high risk of bias fulfilled the inclusion criteria of the present systematic review. Moreover, dissimilar evaluation methods, different outcome measures, 
various methodological confounding factors posed serious restrictions reviewing the literature in a quantitative systematic manner. Hence, the conclusions drawn from the results of this systematic review should be cautiously interpreted and welldesigned long-term randomized controlled trials applying three-dimensional technology, an economic perspective as well as patient-related outcome measures with the different types of distraction appliance are needed before definite conclusions can be provided.

\section{ACKNOWLEDGMENTS AND DISCLOSURE STATEMENTS}

The authors declare that there are no financial or other conflicts of interest related to this publication. We would like to give a special thanks to Conni Skrubbeltrang (Head of Medical Library, Aalborg University Hospital, Aalborg, Denmark) for her assistance with the search strategy. There were no sources of funding for this systematic review.

\section{REFERENCES}

1. de Gijt JP, Vervoorn K, Wolvius EB, Van der Wal KG, Koudstaal MJ. Mandibular midline distraction: a systematic review. J Craniomaxillofac Surg. 2012 Apr;40(3):248-60. [Medline: 21719302] [doi: 10.1016/j.jcms.2011.04.016]

2. Alkan A, Ozer M, Baş B, Bayram M, Celebi N, Inal S, Ozden B. Mandibular symphyseal distraction osteogenesis: review of three techniques. Int J Oral Maxillofac Surg. 2007 Feb;36(2):111-7. [Medline: 17223309] [doi: 10.1016/j.ijom.2006.11.005]

3. Housley JA, Nanda RS, Currier GF, McCune DE. Stability of transverse expansion in the mandibular arch. Am J Orthod Dentofacial Orthop. 2003 Sep;124(3):288-93. [Medline: 12970662] [doi: 10.1016/S0889-5406(03)00450-5]

4. Little RM. Stability and relapse of dental arch alignment. Br J Orthod. 1990 Aug;17(3):235-41. [Medline: 2207055] [doi: 10.1179/bjo.17.3.235]

5. Alexander CD, Bloomquist DS, Wallen TR. Stability of mandibular constriction with a symphyseal osteotomy. Am J Orthod Dentofacial Orthop. 1993 Jan;103(1):15-23. [Medline: 8422026] [doi: 10.1016/0889-5406(93)70099-A]

6. Conley R, Legan H. Mandibular symphyseal distraction osteogenesis: diagnosis and treatment planning considerations. Angle Orthod. 2003 Feb;73(1):3-11. [Medline: 12607849] [doi: 10.1043/0003-3219(2003)0732.0.CO;2]

7. Rosenthal W. Opisthogenie (Mikrogenie). In: Barth JA, editors. [Spezielle Zahn-, Mund- und Kieferchirurgie: Missbildungen, Entzündungen, Geschwülste]. Leipzig; 1951. p. 89-99.

8. Guerrero CA, Bell WH, Contasti GI, Rodriguez AM. Mandibular widening by intraoral distraction osteogenesis. Br J Oral Maxillofac Surg. 1997 Dec;35(6):383-92. [Medline: 9486441] [doi: 10.1016/S0266-4356(97)90712-9]

9. Boccaccio A, Cozzani M, Pappalettere C. Analysis of the performance of different orthodontic devices for mandibular symphyseal distraction osteogenesis. Eur J Orthod. 2011 Apr;33(2):113-20. [Medline: 20709724] [doi: 10.1093/ejo/cjq050]

10. Raoul G, Wojcik T, Ferri J. Outcome of mandibular symphyseal distraction osteogenesis with bone-borne devices. J Craniofac Surg. 2009 Mar;20(2):488-93. [Medline: 19276820] [doi: 10.1097/SCS.0b013e31819b9d2c]

11. Garreau É, Wojcik T, Rakotomalala H, Raoul G, Ferri J. Symphyseal distraction in the context of orthodontic treatment: a series of 35 cases. Int Orthod. 2015 Mar;13(1):81-95. [Medline: 25703076] [doi: 10.1016/j.ortho.2014.12.003]

12. Nadjmi N, Stevens S, Van Erum R. Mandibular midline distraction using a tooth-borne device and a minimally invasive surgical procedure. Int J Oral Maxillofac Surg. 2015 Apr;44(4):452-4. [Medline: 25487564] [doi: 10.1016/j.ijom.2014.11.004]

13. Niculescu JA, King JW, Lindauer SJ. Skeletal and dental effects of tooth-borne versus hybrid devices for mandibular symphyseal distraction osteogenesis. Angle Orthod. 2014 Jan;84(1):68-75. [Medline: 23786596] [doi: $10.2319 / 022213-154.1]$

14. Iseri H, Malkoç S. Long-term skeletal effects of mandibular symphyseal distraction osteogenesis. An implant study. Eur J Orthod. 2005 Oct;27(5):512-7. [Medline: 16043467] [doi: 10.1093/ejo/cji026]

15. Bell WH, Harper RP, Gonzalez M, Cherkashin AM, Samchukov ML. Distraction osteogenesis to widen the mandible. Br J Oral Maxillofac Surg. 1997 Feb;35(1):11-9. [Medline: 9042998] [doi: 10.1016/S0266-4356(97)90003-6]

16. de Gijt JP, van der Wal KG, Kleinrensink GJ, Smeets JB, Koudstaal MJ. Introduction of the "Rotterdam mandibular distractor" and a biomechanical skull analysis of mandibular midline distraction. Br J Oral Maxillofac Surg. 2012 Sep;50(6):519-22. [Medline: 21924532] [doi: 10.1016/j.bjoms.2011.08.007]

17. Carlino F, Pantaleo G, Ciuffolo F, Claudio PP, Cortese A. New Technique for Mandibular Symphyseal Distraction by a Double-Level Anchorage and Fixation System: Advantages and Results. J Craniofac Surg. 2016 Sep;27(6):1469-75. [Medline: 27607116] [doi: 10.1097/SCS.0000000000002831]

18. Savoldelli C, Bouchard PO, Manière-Ezvan A, Bettega G, Tillier Y. Comparison of stress distribution in the temporomandibular joint during jaw closing before and after symphyseal distraction: a finite element study. Int J Oral Maxillofac Surg. 2012 Dec;41(12):1474-82. [Medline: 22771220] [doi: 10.1016/j.ijom.2012.06.005] 
19. Hollis BJ, Block MS, Gardiner D, Chang A. An experimental study of mandibular arch widening in the dog using distraction osteogenesis. J Oral Maxillofac Surg. 1998 Mar;56(3):330-8. [Medline: 9496845] [doi: 10.1016/S0278-2391(98)90110-0]

20. Welch V, Petticrew M, Tugwell P, Moher D, O’Neill J, Waters E, White H; PRISMA-Equity Bellagio group. PRISMAEquity 2012 extension: reporting guidelines for systematic reviews with a focus on health equity. PLoS Med. 2012;9(10):e1001333. [Medline: 23222917] [doi: 10.1371/journal.pmed.1001333]

21. Durham JN, King JW, Robinson QC, Trojan TM. Long-term skeletodental stability of mandibular symphyseal distraction osteogenesis: Tooth-borne vs hybrid distraction appliances. Angle Orthod. 2017 Mar;87(2):246-53. [Medline: 27654627] [doi: $10.2319 / 022916-175.1]$

22. Landes CA, Laudemann K, Sader R, Mack M. Prospective changes to condylar position in symphyseal distraction osteogenesis. Oral Surg Oral Med Oral Pathol Oral Radiol Endod. 2008 Aug;106(2):163-72. [Medline: 18547830] [doi: 10.1016/j.tripleo.2007.12.032]

23. Gunbay T, Akay MC, Aras A, Gomel M. Effects of transmandibular symphyseal distraction on teeth, bone, and temporomandibular joint. J Oral Maxillofac Surg. 2009 Oct;67(10):2254-65. [Medline: 19761921] [doi: 10.1016/i.joms.2009.04.055]

24. de Gijt JP, Gül A, Sutedja H, Wolvius EB, van der Wal KG, Koudstaal MJ. Long-term (6.5 years) follow-up of mandibular midline distraction. J Craniomaxillofac Surg. 2016 Oct;44(10):1576-82. [Medline: 27614544] [doi: 10.1016/i.jcms.2016.06.023]

25. Del Santo M Jr, Guerrero CA, Buschang PH, English JD, Samchukov ML, Bell WH. Long-term skeletal and dental effects of mandibular symphyseal distraction osteogenesis. Am J Orthod Dentofacial Orthop. 2000 Nov;118(5):485-93. [Medline: 11094362] [doi: 10.1067/mod.2000.109887]

26. Ploder O, Köhnke R, Klug C, Kolk A, Winsauer H. Three-dimensional measurement of the mandible after mandibular midline distraction using a cemented and screw-fixated tooth-borne appliance: a clinical study. J Oral Maxillofac Surg. 2009 Mar;67(3):582-8. [Medline: 19231784] [doi: 10.1016/j.joms.2008.06.102]

27. Seeberger R, Kater W, Davids R, Thiele OC, Edelmann B, Hofele C, Freier K. Changes in the mandibular and dentoalveolar structures by the use of tooth borne mandibular symphyseal distraction devices. J Craniomaxillofac Surg. 2011 Apr;39(3):177-81. [Medline: 20708944] [doi: 10.1016/j.jcms.2010.04.005]

28. Malkoç S, Işeri H, Karaman AI, Mutlu N, Küçükkolbaşi H. Effects of mandibular symphyseal distraction osteogenesis on mandibular structures. Am J Orthod Dentofacial Orthop. 2006 Nov;130(5):603-11. [Medline: 17110257] [doi: 10.1016/j.ajodo.2005.02.024]

29. Kewitt GF, Van Sickels JE. Long-term effect of mandibular midline distraction osteogenesis on the status of the temporomandibular joint, teeth, periodontal structures, and neurosensory function. J Oral Maxillofac Surg. 1999 Dec;57(12):1419-25; discussion 1426. [Medline: 10596662] [doi: 10.1016/S0278-2391(99)90723-1]

30. Mommaerts MY, Spaey YJ, Soares Correia PE, Swennen GR. Morbidity related to transmandibular distraction osteogenesis for patients with developmental deformities. J Craniomaxillofac Surg. 2008 Jun;36(4):192-7. [Medline: 18359238] [doi: $10.1016 /$ j.jcms.2007.10.001]

31. de Gijt JP, Gül A, Wolvius EB, van der Wal KGH, Koudstaal MJ. Complications in Mandibular Midline Distraction. Craniomaxillofac Trauma Reconstr. 2017 Sep;10(3):204-7. [Medline: 28751944] [doi: 10.1055/s-0037-1600902]

32. Chung YW, Tae KC. Dental stability and radiographic healing patterns after mandibular symphysis widening with distraction osteogenesis. Eur J Orthod. 2007 Jun;29(3):256-62. [Medline: 17317863] [doi: 10.1093/ejo/cj1088]

33. Braun S, Bottrel JA, Legan HL. Condylar displacement related to mandibular symphyseal distraction. Am J Orthod Dentofacial Orthop. 2002 Feb;121(2):162-5. [Medline: 11840130] [doi: 10.1067/mod.2002.121560]

34. King JW, Wallace JC, Winter DL, Niculescu JA. Long-term skeletal and dental stability of mandibular symphyseal distraction osteogenesis with a hybrid distractor. Am J Orthod Dentofacial Orthop. 2012 Jan;141(1):60-70. [Medline: 22196186] [doi: 10.1016/j.ajodo.2011.06.030]

35. Alkan A, Arici S, Sato S. Bite force and occlusal contact area changes following mandibular widening using distraction osteogenesis. Oral Surg Oral Med Oral Pathol Oral Radiol Endod. 2006 Apr;101(4):432-6. [Medline: 16545704] [doi: 10.1016/j.tripleo.2005.07.017]

36. Duran I, Malkoç S, Işeri H, Tunali M, Tosun M, Küçükkolbaşi H. Microscopic evaluation of mandibular symphyseal distraction osteogenesis. Angle Orthod. 2006 May;76(3):369-74. [Medline: 16637713] [doi: 10.1043/0003-3219(2006)076[0369:MEOMSD]2.0.CO;2]

37. Bayram M, Ozer M, Alkan A. Mandibular symphyseal distraction osteogenesis using a bone-supported distractor. Angle Orthod. 2007 Jul;77(4):745-52. [Medline: 17605491] [doi: 10.2319/070506-274]

38. Malkoç S, Uşümez S, Işeri H. Long-term effects of symphyseal distraction and rapid maxillary expansion on pharyngeal airway dimensions, tongue, and hyoid position. Am J Orthod Dentofacial Orthop. 2007 Dec;132(6):769-75. [Medline: 18068595] [doi: 10.1016/j.ajodo.2005.11.044]

39. Swennen GR, Schutyser F, Barth EL. A new method of 3D cephalometry part I: the anatomic Cartesian 3-D reference system. J craniofac Surg. 2006 Mar;17(2):314-25. [Medline: 16633181] [doi: 10.1097/00001665-200603000-00019] 
40. Lagravère MO, Gordon JM, Guedes IH, Flores-Mir C, Carey JP, Heo G, Major PW. Reliability of traditional cephalometric landmarks as seen in three-dimensional analysis in maxillary expansion treatments. Angle Orthod. 2009 Nov;79(6):1047-56. [Medline: 19852593] [doi: 10.2319/010509-10R.1]

41. van Vlijmen OJ, Bergé SJ, Bronkhorst EM, Swennen GR, Katsaros C, Kuijpers-Jagtman AM. A comparison of frontal radiographs obtained from cone beam CT scans and conventional frontal radiographs of human skulls. Int J Oral Maxillofac Surg. 2009 Jul;38(7):773-8. [Medline: 19369033] [doi: 10.1016/j.ijom.2009.02.024]

42. van Vlijmen OJ, Bergé SJ, Swennen GR, Bronkhorst EM, Katsaros C, Kuijpers-Jagtman AM. Comparison of cephalometric radiographs obtained from cone-beam computed tomography scans and conventional radiographs. J Oral Maxillofac Surg. 2009 Jan;67(1):92-7. [Medline: 19070753] [doi: 10.1016/j.joms.2008.04.025]

43. Garreau E, Bouscaillou J, Rattier S, Ferri J, Raoul G. Bone-borne distractor versus tooth-borne distractor for orthodontic distraction after surgical maxillary expansion: The patient's point of view. Int Orthod. 2016 Jun;14(2):214-32. [Medline: 27155785] [doi: 10.1016/j.ortho.2016.03.013]

44. von Bremen J, Schäfer D, Kater W, Ruf S. Complications during mandibular midline distraction. Angle Orthod. 2008 Jan;78(1):20-4. [Medline: 18193951] [doi: 10.2319/011507-17.1]

45. Verlinden CR, van de Vijfeijken SE, Tuinzing DB, Jansma EP, Becking AG, Swennen GR. Complications of mandibular distraction osteogenesis for developmental deformities: a systematic review of the literature. Int J Oral Maxillofac Surg. 2015 Jan;44(1):44-9. [Medline: 25442740] [doi: 10.1016/j.ijom.2014.09.007]

46. Kewitt GF, Van Sickels JE. Long-term effect of mandibular midline distraction osteogenesis on the status of the temporomandibular joint, teeth, periodontal structures, and neurosensory function. J Oral Maxillofac Surg. 1999 Dec;57(12):1419-25; discussion 1426. [Medline: 10596662] [doi: 10.1016/S0278-2391(99)90723-1]

47. Mommaerts MY, Polsbroek R, Santler G, Correia PE, Abeloos JV, Ali N. Anterior transmandibular osteodistraction: clinical and model observations. J Craniomaxillofac Surg. 2005 Oct;33(5):318-25. [Medline: 16139505] [doi: $10.1016 /$ j.jcms.2005.02.009]

48. Samchukov ML, Cope JB, Harper RP, Ross JD. Biomechanical considerations of mandibular lengthening and widening by gradual distraction using a computer model. J Oral Maxillofac Surg. 1998 Jan;56(1):51-9. [Medline: 9437982] [doi: 10.1016/S0278-2391(98)90916-8]

\section{To cite this article:}

Starch-Jensen T, Kjellerup AD, Blæhr TL.

Mandibular Midline Distraction Osteogenesis with a Bone-borne, Tooth-borne or Hybrid Distraction Appliance: a Systematic Review

J Oral Maxillofac Res 2018;9(3):e1

URL: http://www.ejomr.org/JOMR/archives/2018/3/e1/v9n3e1.pdf

doi: $10.5037 /$ jomr.2018.9301

Copyright (C) Starch-Jensen T, Kjellerup AD, Blæhr TL. Published in the JOURNAL OF ORAL \& MAXILLOFACIAL RESEARCH (http://www.ejomr.org), 30 September 2018.

This is an open-access article, first published in the JOURNAL OF ORAL \& MAXILLOFACIAL RESEARCH, distributed under the terms of the Creative Commons Attribution-Noncommercial-No Derivative Works 3.0 Unported License, which permits unrestricted non-commercial use, distribution, and reproduction in any medium, provided the original work and is properly cited. The copyright, license information and link to the original publication on (http://www.ejomr.org) must be included. 
Appendix 1. PubMed search until the $3^{\text {th }}$ of July, 2018

\begin{tabular}{c|l|c}
\hline ID & \multicolumn{1}{|c}{ Search terms } & Results \\
\hline 26 & Search “Mandible”[Mesh] & 52509 \\
\hline 28 & Search (Mylohyoid Groove* OR lower jaw* OR Mylohyoid Ridge*) & 3189 \\
\hline 29 & Search (mandibular*[Text Word] OR mandible*[Text Word]) & 106487 \\
\hline 30 & $\begin{array}{l}\text { Search ((“Mandible”[Mesh]) OR ((Mylohyoid Groove* OR lower jaw* OR Mylohyoid Ridge*))) OR } \\
((\text { mandibular*[Text Word] OR mandible*[Text Word])) }\end{array}$ & 109815 \\
\hline 32 & Search “Osteogenesis, Distraction”[Mesh] & 4092 \\
\hline 33 & Search osteodistraction*[Text Word] & 135 \\
\hline 34 & Search (osteogenesis distraction*[Text Word] OR distraction osteogenesis[Text Word]) & 4966 \\
\hline 35 & Search distractions osteogenesis[Text Word] & 80 \\
\hline 36 & $\begin{array}{l}\text { Search (((“Osteogenesis, Distraction”[Mesh]) OR osteodistraction*[Text Word]) OR ((osteogenesis } \\
\text { distraction*[Text Word] OR distraction osteogenesis[Text Word]))) OR distractions osteogenesis[Text Word] }\end{array}$ & 4987 \\
\hline 38 & Search (symphyseal[Text Word] OR symphysial[Text Word]) & 923 \\
\hline 39 & Search (Anterior*[Text Word] OR midline*[Text Word]) & 377844 \\
\hline 40 & $\begin{array}{l}\text { Search (((symphyseal[Text Word] OR symphysial[Text Word]))) OR ((Anterior*[Text Word] OR midline*[Text } \\
\text { Word])) }\end{array}$ & 378516 \\
\hline 41 & $\begin{array}{l}\text { Search ((((“"Mandible”[Mesh]) OR ((Mylohyoid Groove* OR lower jaw* OR Mylohyoid Ridge*))) OR } \\
((\text { mandibular*[Text Word] OR mandible*[Text Word])))) AND ((((“Osteogenesis, Distraction”[Mesh]) OR } \\
\text { osteodistraction*[Text Word]) OR ((osteogenesis distraction*[Text Word] OR distraction osteogenesis[Text Word]))) } \\
\text { OR distractions osteogenesis[Text Word])) AND ((((symphyseal[Text Word] OR symphysial[Text Word]))) OR } \\
((\text { Anterior*[Text Word] OR midline*[Text Word]))) }\end{array}$ & 289 \\
\hline
\end{tabular}

Appendix 2. Embase search until the $3^{\text {th }}$ of July, 2018

\begin{tabular}{|c|c|c|}
\hline ID & Search terms & Results \\
\hline 1 & 'mandible'/exp & 46420 \\
\hline 2 & 'mylohyoid groove*':ti,ab,kw OR 'lower jaw*':ti,ab,kw OR 'mylohyoid ridge*':ti,ab,kw & 3503 \\
\hline 3 & mandible*:ti,ab,kw OR mandibular*:ti,ab,kw & 85417 \\
\hline 4 & $\# 1 \mathrm{OR} \# 2 \mathrm{OR} \# 3$ & 100173 \\
\hline 5 & 'distraction osteogenesis'/exp & 4765 \\
\hline 6 & $\begin{array}{l}\text { osteodistraction*:ti,ab,kw OR 'osteogenesis distraction*':ti,ab,kw OR 'distraction osteogenesis':ti,ab,kw } \\
\text { OR 'distractions osteogenesis':ti,ab,kw }\end{array}$ & 4061 \\
\hline 7 & \#5 OR \#6 & 5477 \\
\hline 8 & symphyseal*:ti,ab,kw OR symphysial*:ti,ab,kw & 1047 \\
\hline 9 & anterior*:ti,ab,kw OR midline*:ti,ab,kw & 458341 \\
\hline 10 & \#8 OR \#9 & 459106 \\
\hline 11 & \#4 AND \#7 AND \#10 & 310 \\
\hline
\end{tabular}

Appendix 3. Cochrane Library search until the $3^{\text {th }}$ of July, 2018

\begin{tabular}{c|l|c}
\hline ID & \multicolumn{1}{|c|}{ Search terms } & Results \\
\hline 1 & MeSH descriptor: [Mandible] explode all trees & 1609 \\
\hline 2 & "mylohyoid groove" or "'lower jaw" or "mylohyoid ridge” & 142 \\
\hline 3 & mandible* or mandibular* & 5079 \\
\hline 4 & $\# 1$ or \#2 or \#3 & 5181 \\
\hline 5 & MeSH descriptor: [Osteogenesis, Distraction] explode all trees & 89 \\
\hline 6 & "Osteogenesis Distraction" or "distraction osteogenesis" or osteodistraction* or "distractions osteogenesis" & 152 \\
\hline 7 & $\# 5$ or \#6 & 152 \\
\hline 8 & symphyseal* or symphysial* or anterior or midline & 20826 \\
\hline 9 & $\# 4$ and \#7 and \#8 & 8 \\
\hline
\end{tabular}

